\title{
Risk factors analysis: work-related musculoskeletal disorders among male traffic policemen using high-powered motorcycles
}

\begin{abstract}
ABSTRCT
Introduction

The occupational safety and health issues for police riders and other professional riders are often related to ergonomic hazards and risks. The purpose of this research was to identify the factors that contribute to the health effects in developing work-related musculoskeletal disorders (WMSDs) among male traffic policemen using high-powered motorcycles.

Materials and methods

A cross-sectional study was conducted using 137 police riders. A set of questionnaires including the Standardised Nordic Questionnaire (SNQ) and human vibration meter (Svantek 106) was used in this study.

Results

The prevalence of WMSDs was $67.9 \%$. Multiple logistic regression analysis revealed that the duration of riding a motorcycle $(\mathrm{OR}=0.175,95 \% \mathrm{CI}: 0.052,0.581)$, years of service $(\mathrm{OR}=0.152,95 \% \mathrm{CI}: 0.040,0.567)$, and hand-arm vibration, HAV $(\mathrm{OR}=3.053,95 \% \mathrm{CI}: 1.126$, 8.280) were significant risk factors for the prevalence of WMSDs.

Discussion

The majority of riders reported symptoms of WMSDs within the past 12 months. Riding duration, years of service, and hand-arm vibration (HAV) were found to be the most important risk factors for WMSDs in this rider group. The results highlight that in the context of ergonomic interaction, high-powered motorcycles and the police riders are not a good fit. Hence, further study is needed to improve the safety and health of the police riders.
\end{abstract}

Keyword : Nordic musculoskeletal questionnaire; Occupational factors; Non-occupational factors; Riders; Vibration 